\title{
RELAÇÃO ENTRE OS VALORES DO VCM E DO RDW-CV EM HEMOGRAMAS DE PACIENTES ATENDIDOS NO LABORATÓRIO DE ANÁLISES CLÍNICAS DO HOSPITAL DAS CLÍNICAS EM ITAPERUNA, RJ
}

\author{
Kleuzenir Silva Turbano LEAL, Josileyde Ribeiro Dutra de SOUZA, Juliano Gomes BARRETO* \& Cristiano \\ Guilherme Alves de OLIVEIRA
}

Universidade Iguaçu - Campus V, Itaperuna, Rio de Janeiro, Brasil.

*Autor para correspondência: julianobarreto@ hotmail.com

http://dx.doi.org/10.18571/acbm.086

\section{RESUMO}

Os índices hematimétricos são uma importante ferramenta de auxílio no diagnóstico de diversas enfermidades como nas anemias. Com o objetivo de analisar as correlações de VMC e do RDW-CV entre faixa etária e sexo, bem como avaliar a relação entre o VCM e o aumento ou diminuição do RDW-CV, foi realizada coleta dos dados entre 318 pacientes atendidos no ambulatório do Laboratório de Análises Clínicas do Hospital das Clínicas, para fins de utilidade diagnóstica dos indicadores automatizados de anisocitose diante da presença ou ausência de alteração do VCM. Foram analisados estatisticamente os dados de correlação entre VCM e de RDW-CV entre faixa etária e sexo com o teste F. Já para os grupos de VCM - Macrocítico, Normocítico e Microcítico - com relação ao RDW-CV, utilizou-se a correlação de Pearson e teste t-Studant. Não houve diferença significativa com relação ao sexo para o índice de RDW$\mathrm{CV}$, apenas para o VCM. A análise realizada na determinação do VCM pelas faixas etárias mostrou diferença significativa apenas entre os indivíduos de 18 a 59 anos com os de até 10 anos e os de 11 a 17 anos. Com relação ao RDW-CV todas as relações apresentaram diferença significativa entre si, exceto a estabelecida entre os de 11 a 17 anos com os de 18 a 59 anos. Os testes sobre os grupos de VCM mostrou significativa correlação apenas nos grupos microcítico e macrocítico. No entanto, todos os grupos mostraram resultado significativo no teste t-Studant.

Palavras-chave: Índices hematimétricos; anemia; sangue; eritrócitos.

\begin{abstract}
The red blood cell rates are an important tool to help the diagnosis of several diseases such as anemia. In order to analyze the correlation between MCV and RDW-CV among age and gender, as well as to assess the relationship between MCV and RDW-CV increasing or reduction, it was performed a data collection among 318 patients treated at the Clinical Laboratory Analysis ambulatory of Hospital Clinics, for the diagnostic purpose of the anisocytisis automated indicators before the presence or absence of MCV change. It was statically assessed the correlation data between MCV and RDW-CV among age and gender with the F test. For the MCV groups - Macrocytic, Normocytic and Microcytic - in relation to RDW$\mathrm{CV}$ it was used the Pearson correlation and the Student-t test. There was no significant difference in relation to gender to the RDW-CV rate, only to MCV. The assessment performed in the MCV determination by age showed significant difference only among the subjects between 18 to 59 years old with the subjects up to 10 years old and the ones between 11 to 17 years old. Regarding the RDW-CV, all relationships showed a significant difference among them, except the one established among the subjects between 11 to 17 years old with the ones between 18 to 59 years
\end{abstract}


old. The tests on the MCV showed a significant correlation only in the microcytic and macrocytic groups. However, all groups showed a significant result in the Student-t test.

Keywords: Red Blood Cell rates; anemia; blood; erythrocytes

\section{Introdução}

A eritropoese é o processo de formação dos eritrócitos. Para uma eritropoese eficaz, a medula óssea necessita de muitos precursores como o ferro, cobalto, vitaminas $\left(\mathrm{B}_{12}\right.$, folato, $\mathrm{C}, \mathrm{E}$, $\mathrm{B}_{6}$, tiamina, riboflavina) e hormônios como andrógenos e tiroxina. A deficiência de qualquer um desses fatores pode estar associada à anemia (HOFFBRAND, 2013).

A anemia decorre pela diminuição da concentração de hemoglobina do sangue abaixo dos valores de referência para idade e sexo (JANNINI 1995; GORINA 1996; LORENZI, HOFFBRAND, 2013). Embora os valores de referência variem entre os laboratórios, valores típicos de hemoglobina para definir anemia seriam abaixo de 13,5 g/dL em homens adultos e abaixo de 11,5 g/dL em mulheres adultas. Dos dois anos de idade até a puberdade, hemoglobina abaixo de $11 \mathrm{~g} / \mathrm{dL}$ indicaria anemia. Os recém-nascidos têm nível mais alto de hemoglobina, ao nascimento, 14g/dL é considerado o limite de referência inferior (HOFFBRAND, 2013).

Segundo Gorina (1996) e Lorenzi (2013), as anemias Microcíticas caracterizam-se por eritrócitos de pequeno volume e com baixo conteúdo hemoglobínico ou hipocrômicas.

A deficiência de ferro é a causa mais frequente de anemia em todo o mundo, constituindo um grave problema de saúde pública em nosso meio. Várias diferenças entre a distribuição de tamanhos dos eritrócitos (RDW) têm sido descritas, com maior anisocitose em casos de anemia ferropriva (MELO et al., 2002; NAOUM, 2011). Na anemia Microcítica tanto VCM quanto o HCM estarão diminuídos e a microscopia da distensão de sangue mostra eritrócitos pequenos (microcíticos) e pálidos (hipocrômicos) aspectos que decorrem de defeitos na síntese de hemoglobina (HOFFBRAND, 2013).

De acordo com Gorina (1996) e Lorenzi (2013), as anemias Macrocíticas caracterizam-se por apresentarem hemácias grandes em volume e geralmente com elevado conteúdo hemoglobínico, sendo que dentro das anemias Macrocíticas se enquadram as megaloblásticas.

As anemias Megaloblásticas caracterizam-se por eritroblastos na medula óssea que mostram um atraso na maturação do núcleo em relação ao citoplasma. A síntese defeituosa de RNA é a causa desse defeito, em geral causado pela deficiência de Vitamina B12 ou de folato. É uma anemia macrocítica, sendo os macrócitos ovais, com VCM > 98, podendo chegar em casos severos a 120-140 fL (HOFFBRAND, 2013).

Sob o ponto de vista da análise laboratorial as anemias são classificadas por meio da utilização dos índices hematimétricos de VCM (volume corpuscular médio) e de HCM (hemoglobina corpuscular média) (NAOUM, 2011).

Os hemogramas automatizados avaliam a média do tamanho dos eritrócitos (VCM) através da impedância, impulsos elétricos emitidos pela passagem de cada célula individualmente em um fluxo, cuja intensidade é proporcional ao tamanho das células. Esses pulsos elétricos são posteriormente, convertidos em fentolitros (fL) (MONTEIRO, 2009).

O Volume Corpuscular Médio (VCM) é um parâmetro que representa uma média do tamanho das hemácias utilizado no exame de hemograma. Valores de VCM acima de 100fL indica macrocitose, abaixo de 80fL em adultos microcitose. O VCM deixou de ser um quociente (Hct $\div$ E) para se tornar a média de uma distribuição de valores que pode gerar uma curva de frequência denominada histograma, com seu coeficiente de variação designado Red Cell Distribution Width (RDW) (FAILACE, 2009). 
O RDW (red cell distribuition width) é medido como um coeficiente de variação da distribuição das hemácias, ou seja, é um indicativo do grau de anisocitose das hemácias expresso em porcentagem (GROTTO, 2008). Segundo Rapaport (1996), o RDW é determinado dividindo o desvio padrão do VCM pelo VCM e multiplicando por 100, convertendo-se assim o valor em porcentagem. Sendo assim, o RDW fornece uma medida quantitativa da variação do tamanho dos eritrócitos circulantes, o que determina a anisocitose.

Monteiro (2009) menciona a escassez de estudos sobre o RDW e a sua relação com microcitose ou macrocitose, bem como sobre a presença da elevação desse índice antes da alteração do volume corpuscular médio (VCM), que, por representar uma média do tamanho dos eritrócitos, pode estar normal, mesmo com anisocitose presente (RDW elevado).

É relatado que algumas condições causadoras de microcitose são acompanhadas por uma heterogeneidade do tamanho das hemácias tais como a anemia ferropriva, enquanto outras doenças, como a talassemia menor e a anemia de doença crônica, apresentam homogeneidade da população microcítica, levando a valores de RDW aumentados ou normais, respectivamente (MATOS, 2008). Nunes (2010) em sua pesquisa menciona o RDW como uma ferramenta útil para o diagnóstico de anemias ferroprivas, sendo esse o primeiro índice a se elevar na presença dessa condição.

O RDW-CV normal, nos aparelhos mais utilizados, está entre 11,5 e 14,5\% (FAILACE, 2009).

Objetivou-se com a pesquisa, analisar a relação entre os valores do VCM e do RDW-CV, determinar a relação entre os valores obtidos com relação ao sexo e à faixa etária dos pacientes e avaliar a relação entre o VCM e o aumento ou diminuição do RDW-CV - para fins de utilidade diagnóstica dos indicadores automatizados de anisocitose - diante da presença ou ausência de alteração do VCM.

As referências utilizadas para tabelar os valores coletados dos resultados de hemogramas, para a pesquisa, são baseadas na literatura e ainda nos valores de referência adotados pelo Laboratório de Análises Clínicas do Hospital das Clínicas que utiliza o aparelho automatizado (contador hematológico SDH da Labtest ${ }^{\circledR}$ ).

\section{Materiais e Métodos}

A coleta dos dados foi realizada entre 318 pacientes atendidos no ambulatório do Laboratório de Análises Clínicas do Hospital das Clínicas no período de 02 a 05 de setembro de 2013. As informações como sexo e idade foram obtidas diretamente pela consulta à requisição dos pacientes fornecida pelo Laboratório em questão, contendo nome e data de nascimento.

Os hemogramas foram realizados utilizando-se equipamento automatizado - contador hematológico SDH-20 da Labtest ${ }^{\circledR}$, sendo os valores para VCM de 80 a 96fL; RDW de 12 a 14,5\% e de Hemoglobina com valores entre 12 a $16 \mathrm{~g} / \mathrm{dL}$. A partir dos resultados, pode-se analisar os valores de Hemoglobina, VCM e RDW-CV de cada paciente.

Objetivando-se avaliar a possível relação existente entre o VCM e o índice RDW-CV, os pacientes foram separados pelas seguintes faixas etárias: menor ou igual a 10 anos, de 11 a 17 anos, de 18 a 59 anos e igual ou maior de 60 anos. Também foram comparados os resultados dos pacientes separados por sexo e por grupos de VCM: Macrocítico (VCM > $96 \mathrm{fL}$ ), Normocítico (VCM entre 80 e $96 \mathrm{fL}$ ) e Microcítico (VCM < $80 \mathrm{fL}$ ), em relação às variações existentes com o RDW-CV, segundo os intervalos de referência mencionados acima.

A análise estatística dos dados foi realizada através do programa Microsoft Excel 2010, utilizando-se fórmulas matemáticas disponíveis pelo próprio programa e baseando-se nos valores de referências de cada um dos índices relatados em literatura e no contador hematológico do local da pesquisa. Para comparar os resultados entre sexo e faixa etária foi utilizado o teste F. 
Utilizou-se também o teste de correlação de Pearson para a comparação entre os índices em cada um dos grupos de VCM e t-Student como teste de significância.

\section{Resultados}

\subsection{Valores de VCM e RDW-CV em relação ao sexo}

A tabela 1 representa os valores limites de VCM e RDW-CV para o sexo feminino com 196 pacientes e para o sexo masculino 122 pacientes. De acordo com os valores representados na tabela 2, não houve diferença estatística significativa com relação ao sexo para o índice de RDW-CV, ao contrário do resultado encontrado para o VCM o qual apresentou pequena diferença estatística entre os sexos. Para análise dos dados foi utilizado o teste F.

Tabela 1: Valores de VCM e RDW-CV por sexo entre a população atendida no Laboratório de Análises Clínicas do Hospital das Clínicas.

\begin{tabular}{ccc}
\hline Sexo & VCM (fL) & RDW-CV (\%) \\
\hline Feminino & $67,8-101,9$ & $12-18,5$ \\
Masculino & $69,2-97,1$ & $12,3-17,3$ \\
\hline
\end{tabular}

Tabela 2: Análise estatística de VCM e RDW-CV por sexo entre a população atendida no Laboratório de Análises Clínicas do Hospital das Clínicas.

\begin{tabular}{ccc}
\hline Sexo & F (Fcrítico) VCM & F (Fcrítico) RDW-CV \\
\hline Feminino & $0,81(0,77) *^{\mathrm{a}}$ & $1,30(1,31)^{* \mathrm{~b}}$ \\
Masculino & $0,81(0,77) *^{\mathrm{a}}$ & $1.30(1,31){ }^{* \mathrm{~b}}$ \\
\hline
\end{tabular}

*a: Valor de F e (Fcrítico) respectivamente no teste de significância entre os sexos para VCM; *b: Valor de F e (Fcrítico) respectivamente no teste de significância entre sexos para RDW-CV. Se F>(Fcrítico) = Há diferença significativa entre sexos. Se F<(Fcrítico) = Não há diferença significativa entre os sexos.

\subsection{Valores de VCM e RDW-CV por faixa etária}

Os valores limites de VCM e RDW-CV por faixa etária encontram-se disponíveis na tabela 3. Para a comparação entre esses dados, foi realizado o estudo estatístico (teste F). A partir dessa análise pode-se observar que, com relação ao VCM o grupo de 18 a 59 anos apresentou diferença significativa apenas com os grupos até 10 anos e com os de 11 a 17 anos. As demais relações não obtiveram diferença significativa entre si, conforme se vê na tabela 4 abaixo.

Ao comparar os valores de RDW-CV relacionados na tabela 5, percebeu-se que todas as relações apresentaram diferença significativa entre si, exceto a estabelecida entre os indivíduos de 11 a 17 anos com os de 18 a 59 anos. 
Tabela 3: Valores de VCM e RDW-CV por faixa etária entre a população atendida no Laboratório de Análises Clínicas do Hospital das Clínicas.

\begin{tabular}{clc}
\hline Idade & VCM (fL) & RDW-CV (\%) \\
\hline$\leq 10$ anos & $67,8-98,2$ & $12-17,3$ \\
$11-17$ anos & $70,2-93,4$ & $12,3-15,1$ \\
$18-59$ anos & $68,8-99,3$ & $12,2-18,5$ \\
$\geq 60$ anos & $67,1-101,9$ & $12,4-16$ \\
\hline
\end{tabular}

Tabela 4: Correlação entre os valores de VCM estudados por faixa etária entre a população atendida no Laboratório de Análises Clínicas do Hospital das Clínicas*

\begin{tabular}{lcccc}
\hline & $\leq \mathbf{1 0}$ anos & $\mathbf{1 1}$ a $\mathbf{1 7}$ anos & $\mathbf{1 8}$ a 59 anos & $\geq \mathbf{6 0}$ anos \\
\hline$\leq 10$ anos & - & $1,11(1,84)$ & $0,99(0,66)$ & $1,19(1,56)$ \\
11 a 17 anos & $1,11(1,84)$ & - & $0,89(0,58)$ & $1,07(1,66)$ \\
18 a 59 anos & $0,99(0,66)$ & $0,89(0,58)$ & - & $1,20(1,42)$ \\
$\geq 60$ anos & $1,19(1,56)$ & $1,07(1,66)$ & $1,20(1,42)$ & - \\
\hline$*$ Valor de $\mathrm{F}$ e (Fcrítico) respectivamente. Se F>(Fcrítico $)=$ Há diferença significativa entre as faixas \\
etárias. Se F<(Fcrítico) = Não há diferença significativa entre as faixas etárias.
\end{tabular}

Tabela 5: Correlação entre os valores de RDW-CV estudados por faixa etária entre a população atendida no Laboratório de Análises Clínicas do Hospital das Clínicas*

\begin{tabular}{lcccc}
\hline & $\leq \mathbf{1 0}$ anos & $\mathbf{1 1}$ a $\mathbf{1 7}$ anos & $\mathbf{1 8}$ a 59 anos & $\mathbf{2 6 0}$ anos \\
\hline$\leq 10$ anos & - & $4,13(1,84)$ & $1,58(1,44)$ & $2,94(1,56)$ \\
11 a 17 anos & $4,13(1,84)$ & - & $0,38(0,58)$ & $0,71(0,56)$ \\
18 a 59 anos & $1,58(1,44)$ & $0,38(0,58)$ & - & $1,86(1,42)$ \\
$\geq 60$ anos & $2,94(1,56)$ & $0,71(0,56)$ & $1,86(1,42)$ & - \\
\hline * Valor de $\mathrm{F}$ e (Fcrítico) respectivamente. Se $\mathrm{F}>$ (Fcrítico) $=$ Há diferença significativa entre as faixas etárias. \\
Se F<(Fcrítico) = Não há diferença significativa entre as faixas etárias.
\end{tabular}

\subsection{Correlação entre os índices de VCM e RDW-CV por Grupos}

Ao avaliar a correlação entre o VCM e o RDW-CV no grupo microcítico com total de 48 (quarenta e oito) pacientes, observou-se que 27,1\% apresentou RDW-CV acima dos valores de normalidades estabelecidos tanto na literatura quanto aos valores do contador hematológico utilizado $(14,5 \%)$. A análise estatística obtida pela correlação de Pearson demostrou fraca correlação negativa entre os índices. Segundo o teste t de Student incidido sobre os resultados, observou-se uma significativa correlação entre os valores de VCM e RDW-CV, onde valores menores de VCM correlacionaram significativamente com valores mais altos de RDW-CV (Tabela 6). 
Tabela 6: Correlação entre os índices VCM e RDW-CV no grupo microcítico (VCM < 80 fL) entre a população atendida no Laboratório de Análises Clínicas do Hospital das Clínicas*

\begin{tabular}{ccc}
\hline & VCM & RDW-CV \\
\hline VCM & - & $-0,56(\mathrm{~S})^{* *}$ \\
RDW-CV & $-0,56(\mathrm{~S})^{* * *}$ & - \\
\hline
\end{tabular}

*Resultado representado por $\mathrm{R}$ (índice de correlação). $\mathrm{Se} \mathrm{R}>0$ = correlação positiva; $\mathrm{R}<0$ = correlação negativa; $\mathrm{R}=0$ ou próximo de zero até $\pm 0,10=$ sem correlação. $* *(S)=$ resultado significativo pelo teste $\mathrm{T}$.

O grupo macrocítico totalizou 5 (cinco) pacientes, dos quais $40 \%$ possuíam valores de RDW-CV acima da referência, sendo que os demais indivíduos mantiveram valores de RDW$\mathrm{CV}$ dentro da normalidade. O teste de Pearson informou fraca correlação negativa, com resultado significativo para o teste T entre os índices, de acordo com a tabela 7.

Tabela 7: Correlação entre os índices VCM e RDW-CV no grupo macrocítico (VCM > 96 fL) entre a população atendida no Laboratório de Análises Clínicas do Hospital das Clínicas*

\begin{tabular}{ccc}
\hline & VCM & RDW-CV \\
\hline VCM & - & $-0,23(\mathrm{~S})^{* *}$ \\
RDW-CV & $-0,23(\mathrm{~S})^{* * *}$ & - \\
\hline
\end{tabular}

*Resultado representado por $\mathrm{R}$ (índice de correlação). $\mathrm{Se} \mathrm{R}>0$ = correlação positiva; $\mathrm{R}<0$ = correlação negativa; $\mathrm{R}=0$ ou próximo de zero até $\pm 0,10=$ sem correlação. $* * \mathrm{~S}=$ resultado significativo pelo teste $\mathrm{T}$.

A tabela 8 apresenta o valor de correlação encontrado no grupo normocítico com total de 265 (duzentos e sessenta e cinco) pacientes, onde 6,79\% apresentaram valor de RDW-CV acima dos valores de referência. O estudo, apesar de positivo, não mostrou correlação entre os índices analisados. No entanto, observou-se resultado significativo no teste T.

Tabela 8: Correlação entre os índices VCM e RDW-CV no grupo normocítico (VCM 80 96 fL) entre a população atendida no Laboratório de Análises Clínicas do Hospital das Clínicas*

\begin{tabular}{ccc}
\hline & VCM & RDW-CV \\
\hline VCM & - & $0,08(\mathrm{~S}) * *$ \\
RDW-CV & $0,08(\mathrm{~S}) * *$ & - \\
\hline *Resultado representado por R (índice de correlação). Se $\mathrm{R}>0$ = correlação positiva; $\mathrm{R}<0=$ correlação \\
negativa; R=0 ou próximo de zero até $\pm 0,10=$ sem correlação. $* * \mathrm{~S}=$ resultado significativo pelo teste T.
\end{tabular}

\section{Discussão}

De acordo com os dados expostos acima para a análise entre os sexos, a presente pesquisa não mostrou, estatisticamente, diferença para o índice RDW-CV. O VCM apresentou pequena diferença estatística entre os sexos, sendo esse resultado diferente dos dados encontrados na pesquisa realizada por Monteiro (2009) com 306 pacientes, o qual não encontrou diferença estatística entre os sexos.

Monteiro (2009) relata ter encontrado diferença entre todas as faixas etárias analisadas em sua pesquisa, exceto daquela entre os indivíduos de 7 a 10 anos com os de 11 a 17 anos ao comparar o VCM. Já os resultados dessa pesquisa mostrou que com relação ao VCM, o grupo de 18 a 59 anos apresentou diferença significativa apenas com os grupos até 10 anos e de 11 a 17 anos, não obtendo diferença significativa das demais relações entre si.

No estudo feito com o RDW-CV pode-se notar que excetuando a relação estabelecida entre os indivíduos de 11 a 17 anos com os de 18 a 59 anos, todas as relações apresentaram 
diferença significativa entre si, enquanto na pesquisa de Monteiro (2009), houve diferença significativa entre as faixas etárias de menores de 6 anos com os de 7 a 10 anos e dos de 7 a 10 anos com os de 11 a 17 anos. O autor menciona ainda que as faixas etárias de 18 a 59 anos e os maiores de 60 anos não apresentaram diferença significativa entre si.

Correlacionando os valores entre o VCM e o RDW-CV, observou-se que $27,1 \%$ dos pacientes apresentaram RDW-CV acima dos valores de normalidades preconizados no trabalho, onde a correlação de Pearson demostrou fraca correlação negativa entre os índices. Ao teste t de Student incidido sobre os resultados, mostrou-se uma significativa correlação, onde valores menores de VCM correlacionaram significativamente com valores mais altos de RDW-CV. Em pesquisa com 159 pacientes portadores de anemia ferropriva, anemia de doença crônica e $\beta$ talassemia, Matos et al. (2008) afirma não ter observado diferença significativa para o RDW-CV entre esses grupos, não sendo esse índice ferramenta útil para o diagnóstico das condições mesmo encontrando leve tendência de elevação de RDW na anemia ferropriva - por não apresentarem diferença estatística significativa entre os grupos. Já Caporal (2013) analisando os índices de RDW-CV, RDW-SD e MATH-1SD em 806 amostras trabalhando com grupos microcíticos, normocíticos e macrocíticos, menciona que o RDW-CV teve maior sensibilidade $(86,8 \%)$ ao detectar anisocitose em amostras microcíticas. O teste de correlação feito por Monteiro (2009) mostrou que valores menores de VCM se correlacionam significativamente com valores maiores de RDW-CV. Almeida et al (2006) justifica a importância clínica do RDW por ser a alteração laboratorial mais precoce de carência de ferro - o que pressupõe um aumento de RDW para níveis mais baixos de VCM - concluindo o trabalho ao dizer que a interpretação conjunta do VCM com o RDW tem indiscutível utilidade no diagnóstico dessas anemias.

Monteiro (2009) relata que 39,28\% dos indivíduos do grupo macrocítico apresentaram valores de RDW-CV acima dos valores de normalidade, bem parecido com o grupo macrocítico da presente pesquisa, dos quais $40 \%$ obtiveram elevação do índice. Porém, o autor mostrou não haver correlação em sua análise estatística de VCM com RDW-CV no grupo macrocítico, diferentemente desse trabalho, o qual apresentou fraca correlação negativa, com resultado significativo para o teste $\mathrm{T}$ entre os índices.

No grupo normocítico com 265 pacientes, 6,79\% apresentaram valor de RDW-CV acima dos valores de referência. Monteiro (2009) obteve resultados de 22,65\% de RDW-CV elevado nos pacientes com normocitose, encontrando baixa correlação entre VCM e RDW-CV nesse grupo, com significativa diferença estatística para o teste T. Embora a presente pesquisa não tenha mostrado correlação para esse grupo, os resultados estatísticos foram significativos pelo teste T, assim como na pesquisa realizada por Monteiro (2009).

\section{Conclusão}

De acordo com a análise de dados realizada, pode-se observar que para o VCM houve pequena diferença estatística entre os sexos, não sendo encontrada diferença estatística significativa para o índice de RDW-CV.

Analisando as faixas etárias, concluiu-se que houve diferença significativa com relação ao VCM no grupo de 18 a 59 anos com os grupos até 10 anos e de 11 a 17 anos, não obtendo diferença significativa as demais relações. Diferente dos valores de RDW-CV onde pode-se perceber que todas as relações apresentaram diferença significativa entre si, exceto a estabelecida entre os indivíduos de 11 a 17 anos com os de 18 a 59 anos.

A correlação entre VCM e RDW-CV o grupo microcítico mostrou significativa correlação entre os índices, demostrando valores menores de VCM correlacionando significativamente com valores mais altos de RDW-CV. O grupo macrocítico apresentou fraca correlação negativa, com resultado significativo para o teste $\mathrm{T}$ entre os índices e o grupo 
normocítico não mostrou correlação entre os índices analisados. Os resultados da pesquisa sugerem que há correlação entre os índices e dessa forma, bem como nos resultados encontrados por outros autores citados, podem ser levados em consideração para utilidade diagnóstica das anemias em especial a microcítica.

\section{Referências}

ALMEIDA, S. Importância clínica do RDW na criança com anemia. Saúde Infantil, Coimbra, v. 2, n. 28, p.7-11, 2006.

ASLAN, D. et al. Importance of RDW value in differential diagnosis of hypochrome anemias. American Journal Of Hematology. Turquia, p. 31-33. jan. 2002.

CAPORAL, F. A. Evaluation of RDW-CV, RDW-SD, and MATH-1SD for the detection of erythrocyte anisocytosis observed by optical microscopy. J. Bras. Patol. Med. Lab. Rio de Janeiro, p. 324-331. out. 2013.

DALANHOL, M. et al. Efeitos quantitativos da estocagem de sangue periférico nas determinações do hemograma automatizado. Revista Brasileira de Hematologia e Hemoterapia, Cascavel, n. , p.1-7, 04 ago. 2009.

FAILACE, R. et al. Hemograma manual de interpretações. 5. ed. Porto Alegre: Artmed, 2009. $424 \mathrm{p}$.

FATEMI, O. et al. Red cell distribution width as a bleeding predictor afterpercutaneous coronary intervention. American Heart Journal. Washington, p. 104-109. 20 maio 2013.

FARIAS, M. G.; BÓ, S. D. Importância clínica e laboratorial do volume plaquetário médio. J Bras Patol Med Lab, Porto Alegre, p. 275-281. 20 ago. 2010.

FRANÇA, M. G. G. Prevalência de Anemia Ferropriva em adolescentes da "Vila Princesa" - Lixão em Porto Velho-RO. 2006. 75 f. Dissertação (Mestrado) - Curso de Ciências da Saúde, Faculdade de Ciências da Saúde da Universidade de Brasília, Porto Velho, 2006.

GORINA, A. B. A Clínica e o Labortório. 16 ed. Barcelona: Medsi, 1996. 556 p.

GROTTO, H. Z. W. Diferenciação das anemias microcíticas utilizando a determinação do RDW. Rev. Bras. Hematol. Hemoter. 2008, vol.30, n.2, p. 87-88.

HADLER, M. C. C.m.; JULIANO, Y.; SIGULEM, D. M.. Anemia do lactente: etiologia e prevalência. Jornal de Pediatria. Rio de Janeiro, p. 321-326. ago. 2002.

HOFFBRAND, V. A.; MOSS. H. A. P. Fundamentos em Hematologia. 6. ed. Porto Alegre: Artmed, 20013. 462 p.

JANNINI, P.; JANNINI FILHO, P. Interpretação Clínica do Hemograma. 10. ed. São Paulo: Sarvier, $1995.625 \mathrm{p}$.

LEE, G. R. et al. Wintrobe Hematologia Clínica. São Paulo: Manole Ltda, 1998. 1424 p. 
LIMA, C. S. P. et al. Comparison of red cell distribution width ad a red cell discriminant function incorporating volume dispersion for distinguishing iron deficiency from beta thalassemia trait in patients with microcytosis. São Paulo Medical Journal. São Paulo, p. 12651269. jan. 1996.

LORENZI, T. F. Manual de Hematologia: Propedêutica e Clínica. 4. ed. Rio de Janeiro: Guanabara, 2013. 710 p.

MATOS, J. F. et al. O hemograma nas anemias microcíticas e hipocrômicas: aspectos diferenciais. J Bras Patol Med Lab, Belo Horizonte, p. 255-258. 20 ago. 2012.

MATOS, J. F. et al. Índice de anisocitose eritrocitária (RDW): diferenciação das anemias microcíticas e hipocrômicas. Rev. Bras. Hematol. Hemoter. 2008, vol.30, n.2, p. 120-123.

MATOS, J. F. et al. $463 \mathrm{O}$ papel do RDW, da morfologia eritrocitária e de parâmetros plaquetários na diferenciação entre anemias microcíticas e hipocrômicas. Revista Brasileira de Hematologia e Hemoterapia, Belo Horizonte, v. 6, n. 30, p.463-469, 29 ago. 2008.

MELO, M. R. Uso de índices hematimétricos no diagnóstico diferencial de anemias microcíticas: uma abordagem a ser adotada? Rev Assoc Med Bras, São Paulo, p.222-224, 1 jan. 2002.

MONTEIRO, L. Valores de referência do RDW-CV e do RDW-SD e sua relação com o VCM entre os pacientes atendidos no ambulatório do Hospital Universitário Oswaldo Cruz - Recife, PE. Revista Brasileira de Hematologia e Hemoterapia, Recife, p.1-6, 27 maio 2009.

NUNES, M. F. P.; ASSIS, A. M. O.; PINHEIRO, S. M. C. e MEDRADO, F. E. R. Erythrocyte indices and serum ferritin in newborns. Rev. Bras. Hematol. Hemoter. 2010, vol.32, n.5, p.365-370.

RAPAPORT, S. I. Hematologia: Introdução. 2. ed. São Paulo: Roca Ltda, 1990. 450 p.

SGNAOLIN, V. et al. Hematological parameters and prevalence of anemia among freeliving elderly in south Brazil. Rev. Bras. Hematol. Hemoter. 2013, vol.35, n.2, p.115-118.

ZAGO, M. A. et al. Hematologia: Fundamentos e Prática. São Paulo: Atheneu, 2004. 1081 p. 